\title{
Impacts of Energy Reconstruction on SBN Oscillation Measurements
}

LeRayah Neely-Brown, Purdue University

Fernanda Psihas \& Joseph Zennamo, Fermilab

\section{Background}

Short Baseline Neutrino (SBN) Program main goal is to measure how neutrinos change into different flavors throughout the universe. Experiments within the SBN Program have seen oscillations beyond expected.

This begs the question, are some oscillations anomalies or show something concrete?

Ultimately, studying oscillations can help us determine if sterile neutrinos, the potential fourth neutrino, are present.

\section{Purpose}

In this project, we analyzed how energy reconstruction within the SBND \& ICARUS detectors can allow us to have improved searches for understanding how neutrinos oscillate and change from one flavor to another.

\section{Materials and Methods}

With the use of SBND and ICARUS monte carlo and the Common Analysis Framework, total energy plots, total shower energy plots, and total calorimetric track energy plots were created to complete precise reconstructed energies to help detect oscillations.

\section{$P_{\text {눈 }}$

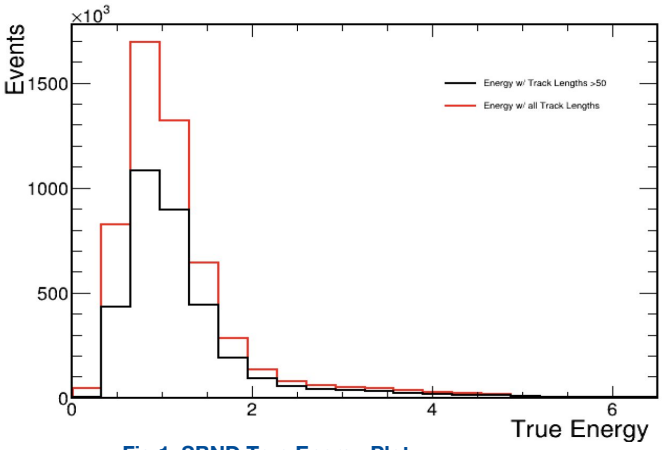

Fig.1: SBND True Energy Plot

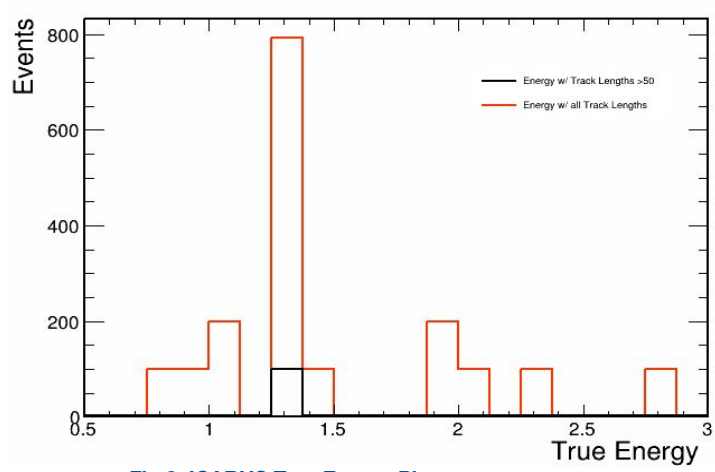

Fig.2: ICARUS True Energy Plot
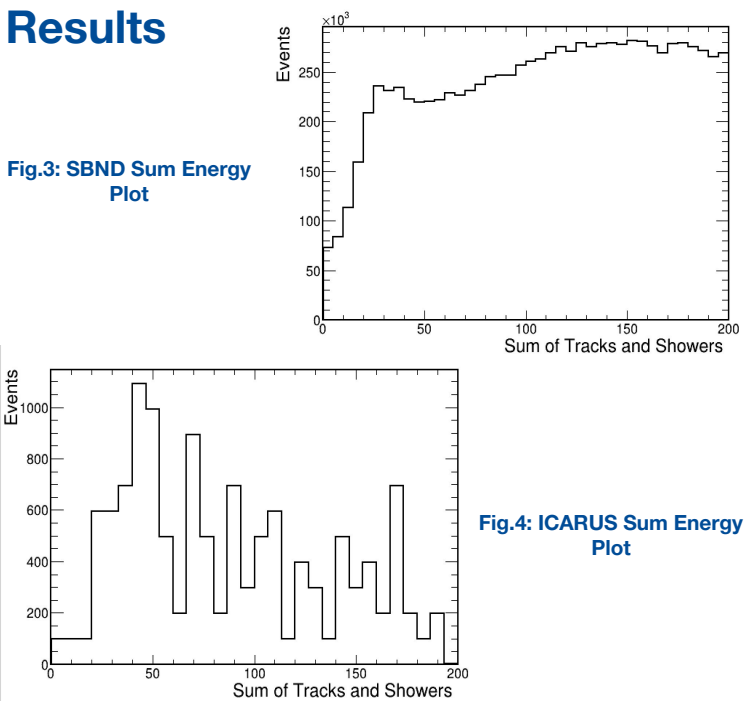

The True Energy of both SBND and ICARUS were used to be plotted against the sum of energy for the tracks and showers of both detectors to estimate and analyze how much the energy for these detectors must be reconstructed to detect neutrino oscillations.

\section{Conclusions}

From these specific studies, we were able to conclude the correlation between the track and shower energies versus the total energies allows for us to have improved searches for oscillations. 